\title{
Genetic Polymorphism of MDM2 SNP309 in Patients with Helicobacter Pylori-Associated Gastritis
}

\author{
Taweesak Tongtawee ${ }^{1,4 *}$, Chavaboon Dechsukhum ${ }^{3,4}$, Wilairat Leeanansaksiri ${ }^{5}$ \\ Soraya Kaewpitoon ${ }^{2,4}$, Natthawut Kaewpitoon ${ }^{5,6}$, Ryan A Loyd ${ }^{2,4}$, Likit \\ Matrakool $^{1,4}$, Sukij Panpimanmas ${ }^{1,4}$
}

\begin{abstract}
Background: Helicobacter pylori plays an important role in gastric cancer, which has a relatively low inciduence in Thailand. MDM2 is a major negative regulator of $\mathbf{p 5 3}$, the key tumor suppressor involved in tumorigenesis of the majority of human cancers. Whether its expression might explain the relative lack of gastric cancer in Thailand was assessed here. Materials and Methods: This single-center study was conducted in the northeast region of Thailand. Gastric mucosa from 100 patients with Helicobacter pylori associated gastritis was analyzed for MDM2 SNP309 using real-time PCR hybridization (light-cycler) probes. Results: In the total 100 Helicobacter pylori associated gastritis cases the incidence of SNP 309 T/T homozygous was $78 \%$ with SNP309 G/T heterozygous found in $19 \%$ and SNP309 G/G homozygous in 3\%. The result show SNP 309 T/T and SNP 309 G/T to be rather common in the Thai population. Conclusions: Our study indicates that the MDM2 SNP309 G/G homozygous genotype might be a risk factor for gastric cancer in Thailand and the fact that it is infrequent could explain to some extent the low incidence of gastric cancer in the Thai population.
\end{abstract}

Keywords: MDM2 SNIP 309 - Helicobacter pylori - gastric cancer

Asian Pac J Cancer Prev, 16 (16), 7049-7052

\section{Introduction}

MDM2 (mouse double minute 2 homolog) is the major negative regulator of $\mathrm{p} 53$, the key tumor suppressor involved in the tumorigenesis of the majority of most human cancer. MDM2 is proposed to regulate p53 at post-translational level by enhancing of p53 degradation through E3 ligase activity (Haupt, et al., 1997, Honda et al., 1997; Kubbutat, et al., 1997). Moreover, MDM2 is also able to inhibit the function of pRB and subsequently increase the level of free E2F1 (Xiao et al., 1995). In addition, MDM2 also bind directly to E2F1 and enhance its activity as a transcription factor (Martin, et al., 1995). According to these cellular functions, MDM2 behaves as an oncoprotein, which has been supported by both in in vitro and clinical studies. Increased levels of MDM2 have been detected in several human cancers. Four mechanisms underlying the overexpression of MDM2 have been proposed. The two major mechanisms are gene amplification (Oliner et al., 1992; Momand et al., 1998) and enhanced translation (Sheikh, et al., 1993; Landers et al., 1997; Trotta et al., 2003) both of which have been shown in several cancers with wild-type TP53. The third mechanism is due to the altered MDM2 degradation which is induced by Casein phosphorylation with subsequently
SCF $\beta$-TRCP ubiquitin ligase (Inuzuka et al., 2010). This scenario is supported by the finding that $\beta$-TCRP deletions are found in several cancers (Momand et al., 2000; Rayburn et al., 2005; Inuzuka, et al., 2010). The forth mechanism of MDM2 hyperactivity is proposed to be due to the genetic polymorphism at MDM2 promoter area located in intron 1 (intronic promoter) (Bond et al., 2004). There are two types of single nucleotide polymorphism (SNPs), which is attributable to MDM2 gene expression level. The MDM2 SNP309 and MDM2 SNP285 are located at position 309 and $285 \mathrm{bp}$ downstream of MDM2 exon 1.These two areas of SNPs are the positions where Sp1 interact with the intronic promoters albeit with different binding activities. SNP309 is shown to enhance Sp1 binding therefore promoting the MDM2 expression. On the other hand, SNP285 decrease the binding of Sp1 to the promoter region (Knappskog et al., 2011). For this reason, MDM2 SNP309 is proposed to increase cancer risk (Bond et al., 2006; Hu et al., 2007; Economopoulos et al., 2010; Post et al., 2010). This hypothesis has been supported by several clinical studies in various human cancers, whereas MDM2 SNP285 has been found to provide the protective effect on human cancers (Hu et al., 2007; Knappskog et al., 2011).

The clinical data concerning the role of MDM2

${ }^{1}$ Department of Surgery, ${ }^{2}$ Family Medicine and Community Medicine, ${ }^{3}$ Pathological Unit, Institute of Medicine, ${ }^{5}$ Parasite Research Unit and Institute of Science, Suranaree University of Technology, ${ }^{6}$ Faculty of Public Health, Vongchawalitkul University, ${ }^{4}$ Suranaree University of Technology Hospital, Nakhon Rachasima, Thailand *For correspondence: taweesak.t@sut.ac.th 
SNP309 in gastric cancer development is limited. The case-control study among the Iranian gastric cancer population showed that MDM2 SNP309 is the risk factor for this cancer with Odds ratio $=2.08(95 \%$ Confident interval=1.37-4.34) (Moradi et al., 2014).

The similar study in Chinese gastric cancer population also showed the same trend (Wang et al., 2009). We are interested in pursuing the clinical study in Thai population to evaluate the possible association between MDM2 SNP309 and gastric cancer risk. As inflammatory processed is also influenced by p53, we also evaluate the possible association between this SNP and the degree of chronic gastritis associated with Helicobacter pylori, which is the precursor lesion for gastric cancer.

\section{Materials and Methods}

\section{Patients}

A total of 100 gastric tissues from the patients diagnosed with Helicobacter pylori associated gastritis proved by positive histology that participated in this study from November 2014 through June 2015. The study was performed in accordance with good clinical practice and the guidelines of the Declaration of Helsinki. All patients provided written informed consent and the study protocol was approved by the Ethics Committee for Research Involving Human Subjects, Suranaree University of Technology (EC-57-34).

\section{Histological analysis}

Gastric tissue specimens for histological analysis were sent to the pathologist. The hematoxylin and eosin stain and Giemsa stain were used for identification of Helicobacter pylori. The pathological analysis was made by 5 pathologists at Bangkok Pathological Laboratory outside of Suranaree University of Technology.

\section{DNA isolation method}

The DNA of Helicobacter pylori was extracted from frozen gastric tissue biopsy specimens which were stored at a temperature of less than $-20^{\circ} \mathrm{C}$ using the QIAamp DNAFFPE tissue kit (Qiagen, USA). The DNA extraction was performed according to the manufacturer protocol. Briefly, ten tissue sections of $5 \mu \mathrm{M}$ thick were collected in $1.5 \mathrm{ml}$ micro centrifuge tubes. The tissue specimens were placed in a microcentrifuge tube, and buffer ATL (180 $\mu \mathrm{L})$ and proteinase $\mathrm{K}(20 \mu \mathrm{L})$ were added. The samples were mixed by vortexing and incubated at $56^{\circ} \mathrm{C}$ until the tissues were completely lysed. Buffer AL $(200 \mu \mathrm{L})$ was added to the samples, which were subsequently incubated at $70^{\circ} \mathrm{C}$ for 10 minutes. Next, $240 \mu \mathrm{L}$ of $100 \%$ ethanol was added to the samples, which were mixed by vortexing for 15 seconds. Each sample was placed in a QIAamp spin column and centrifuged at $8000 \mathrm{rpm}$ for 1 minute. The columns were washed with AW1 buffer $(500 \mu \mathrm{L})$, and samples were centrifuged at $8000 \mathrm{rpm}$ for 1 minute. AW2 buffer $(500 \mu \mathrm{L})$ was added to the column, and samples were centrifuged at 14,000 rpm for 3 minutes. Buffer AE $(200 \mu \mathrm{L})$ was added to each sample, and samples were incubated for 1 minute prior to centrifugation at $8000 \mathrm{rpm}$ for 1 minute. Finally, DNA was extracted from the tissue.

\section{Polymorphism analysis}

MDM2 SNP309 genotypes were analyzed using real-time PCR. The hybridization probes (light-cycler probe) were utilized for this analysis. The real-time PCR procedure was accomplished by using LightCycler® 480 instrument (Roche diagnostics, Neuilly sur Seine, France). The identifications of target PCR products were accomplished by melting curve analyses. The target PCR products were amplified by using the primers as previously reported in the previous literature. The hybridization probes include the one that is in the SNP309 (the sensor probe). This sensor probe is labeled by LC-red 640 at 5' and phosphorylated at 3'. The anchor probe will hybridized to the PCR product at the site $3 \mathrm{bp}$ upstream to the sensor probe. $3 \mu 1$ DNA templates were subjected to $\mathrm{PCR}$ reaction in the final volume of $20 \mu 1$. The reaction mixture consists of $\mathrm{MgCl} 2(25 \mathrm{mM})$, forward and reverse primers (20 M each), sensor and anchor probes (20 M each), and $2 \mu 1$ of FastStart DNA Master Hybridization Probes (Roche Diagnostics). PCR amplification comprised an initial denaturation cycle at $95^{\circ} \mathrm{C}$ for $10 \mathrm{~min}$, followed by 50 amplification cycles (with a temperature transition rate of $20^{\circ} \mathrm{C} / \mathrm{s}$ ) consisting of $95^{\circ} \mathrm{C}$ for $0 \mathrm{~s}$, annealing at $60^{\circ} \mathrm{C}$ for $10 \mathrm{~s}$, and extension at $72^{\circ} \mathrm{C}$ for $17 \mathrm{~s}$. After amplification a melting step was performed, consisting of $95^{\circ} \mathrm{C}$ for $0 \mathrm{~s}$, cooling to $45^{\circ} \mathrm{C}$ for $30 \mathrm{~s}$ (with a temperature transition rate of $20^{\circ} \mathrm{C} / \mathrm{s}$ ), and finally a slow rise in the temperature to $85^{\circ} \mathrm{C}$ at a rate of $0.1^{\circ} \mathrm{C} / \mathrm{s}$ with continuous acquisition of fluorescence decline. The genotype of each patient can be categorized into three genotypes SNP309 G/G homozygous, T/T homozygous and G/T heterozygous based on the different melting curve.

\section{Statistical analysis}

SPSS for Windows (version 16.0; SPSS, Chicago, IL, USA) was used for the statistical analysis; baseline demographic data of the infected patients were analyzed. The patterns of genetic polymorphism were analyzed by using LightCycler ${ }^{\circledR} 480$ instrument (Roche diagnostics, Neuilly sur Seine, France).

\section{Results}

A total of 100 gastric tissues from Helicobacter pylori associated gastritis patients enrolled into the study. The patients' demographic data are summarized in Table 1 and pattern of MDM2 SNIP309 polymorphism are summarized in Figure 1. The incidence of SNP 309 T/T homozygous was $79 \%$ (79/100), SNP309 G/T heterozygous $18 \%$ $(18 / 100)$ and SNP309 G/G homozygous 3\% (3/100).

\section{Table 1. The Patient Demographic Data}

\begin{tabular}{lr}
\hline Demographics & $\mathrm{n}=100$ \\
\hline Male/female (n) & $24 / 76$ \\
Mean age (years) & 48.2 \\
Peptic ulcer disease (GU/DU) & $15 \%$ \\
Gastric cancer & $1 \%$ \\
Non ulcer gastritis/duodinitis & $84 \%$ \\
SNP 309 T/T & $78 \%$ \\
SNP309 G/T & $19 \%$ \\
MDM2 SNP309 G/G & $3 \%$ \\
\hline
\end{tabular}




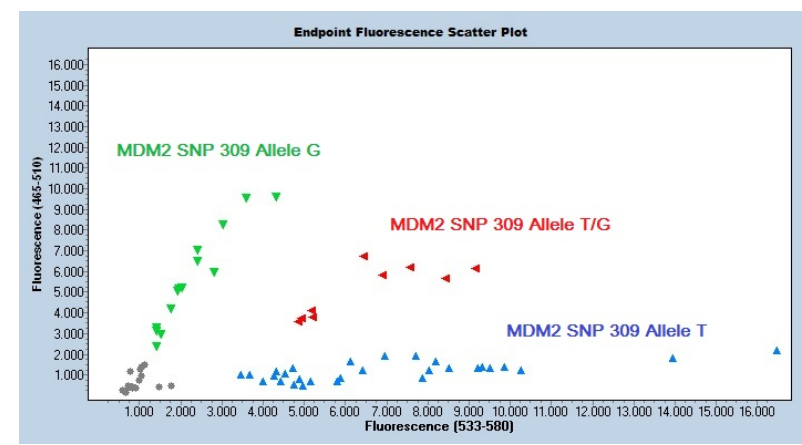

Figure 1. Pattern of Genetic MDM2 SNIP 309 Polymorphism Using Real-time PCR Hybridization Probes (light-cycler probe)

\section{Discussion}

Genetic polymorphism is obviously the one of underlying mechanism, which is attributable to the ethnic difference in cancer predisposition. Single nucleotide polymorphism (SNP) of genomic DNA has been shown to be associated with the risk of various diseases including human cancers. This association obtained from epidemiological data is further supported by in vitro functional analysis and animal model. It becomes clear that some of these SNPs influence the biological function of the cells through various direct or indirect mechanism including gene expression control, at both RNA and protein level, protein structural modification. Therefore, the SNPs genotyping becomes the hot topic for researchers, which hope to identify the individual genetic background applicable to disease prevention, optimized medical treatment and surveillance.

Gastric cancer is the fifth most common human cancers worldwide. Intriguingly, marked variation in the gastric cancer incidence is observed. The highest incidence of gastric cancer is found in Korea $(41.8 / 100,000)$ whereas the lowest incidence is found in Africa and Northern America. In Thailand the incidence of gastric cancer is $3.5 / 100,000$. As the chronic gastritis is the major predisposing condition to gastric cancer, the association study between these conditions has become the area of interest. However, in Thailand where gastric cancer in incidence falls into the low-risk country group, the prevalence of Helicobacter pylori associated gastritis is intriguingly high.

This study aims to investigation the prevalence and patterns of genetic polymorphism of MDM2 gene (MDM2 SNP309) in Thai population. Our data indicate that the incidence of SNP 309 T/T homozygous and SNP309 G/T heterozygous is rather high. According to previous data, these genotypes confer the protective effect for certain human cancers including gastric cancer and may be answer of Thailand enigma.

In conclusion, results of our study indicated that frequency of MDM2 SNP309 G/G is very low, that can explain to some extent the low incidence of gastric cancer in Thai population. According to literature review, this study is the first attempt to evaluate the possible impact of genetic background, MDM2 SNP309 on the gastric cancer in patients with Helicobacter pylori associated gastritis in Thai population.

\section{References}

Bond GL, Menin C, Bertorelle R, et al (2006). MDM2 SNP309 Accelerates colorectal tumour formation in women. $\mathrm{J} \mathrm{Med}$ Genet, 43, 950-2.

Bond GL, Hu W, Bond EE, et al (2004). A single nucleotide polymorphism in the MDM2 promoter attenuates the p53 tumor suppressor pathway and accelerates tumor formation in humans. Cell, 119, 591-602.

Bond GL, Hirshfield KM, Kirchhoff T, et al (2006). MDM2 SNP309 accelerates tumor formation in a gender-specific and hormone-dependent manner. Cancer Res, 66, 5104-10.

Economopoulos KP, Sergentanis TN (2010). Differential effects of MDM2 SNP309 polymorphism on breast cancer risk along with race: a meta-analysis. Breast Cancer Res Treat, 120, 211-6.

Haupt Y, Maya R, Kazaz A, Oren M (1997). Mdm2 promotes the rapid degradation of p53. Nature, 387, 296-9.

Honda R, Tanaka H, Yasuda H (1997). Oncoprotein MDM2 is ubiquitin ligase E3 for tumor suppressor p53. FEBS Lett, 420, 25-7.

$\mathrm{Hu} \mathrm{Z}$, Jin G, Wang L, et al (2007). MDM2 promoter polymorphism SNP309 contributes to tumor susceptibility: evidence from 21 case-control studies. Cancer Epidemiol Biomarkers Prev, 16, 2717-23.

Inuzuka H, Tseng A, Gao D, et al (2010). Phosphorylation by casein kinase I promote the turnover of the Mdm2 oncoprotein via the SCF (beta-TRCP) ubiquitin ligase. Cancer Cell, 18, 147-59.

Inuzuka H, Fukushima H, Shaik S, et al (2010). Novel insights into the molecular mechanisms governing mdm2 ubiquitination and destruction. Oncotarget, 1, 685-90.

Kubbutat MH, Jones SN, Vousden KH (1997). Regulation of p53 stability by Mdm2. Nature, 387, 299-303.

Knappskog S, Bjornslett M, Myklebust LM, et al (2011). The MDM2 promoter SNP285C/309G haplotype diminishes $\mathrm{Sp} 1$ transcription factor binding and reduces risk for breast and ovarian cancer in Caucasians. Cancer Cell, 19, 273-82.

Knappskog S, Trovik J, Marcickiewicz J, et al (2011). Impact of the MDM2 promoter polymorphsims SNP285 and SNP309 on endometrial cancer risk among Caucasians. ASCO, 5092.

Landers JE, Cassel SL, George DL (1997). Translational enhancement of mdm2 oncogene expression in human tumor cells containing a stabilized wild-type $\mathrm{p} 53$ protein. Cancer Res, 57, 3562-8.

Martin K, Trouche D, Hagemeier C, et al (1995). Stimulation of E2F1/DP1 transcriptional activity by MDM2 oncoprotein. Nature, 375, 691-4.

Momand J, Jung D, Wilczynski S, Niland J (1998). The MDM2 gene amplification database. Nucleic Acids Res, 26, 3453-9.

Momand J, Wu HH, Dasgupta G (2000). MDM2-master regulator of the p53 tumor suppressor protein. Gene, $\mathbf{2 4 2}$, 15-29.

Moradi MT, Salehi Z, Aminian K, et al (2014). Effects of p53 codon 72 and MDM2 SNP309 polymorphisms on gastric cancer risk among the Iranian population. Asian Pac J Cancer Prev, 15, 7413-7.

Oliner JD, Kinzler KW, Meltzer PS, et al (1992). Amplification of a gene encoding a 53 -associated protein in human sarcomas. Nature, 358, 80-83.

Post SM, Quintas-Cardama A, Pant V, et al (2010). A high-frequency regulatory polymorphism in the p53 pathwayaccelerates tumor development. Cancer Cell, 18, 220-30.

Rayburn E, Zhang R, He J, et al (2005). MDM2 and human malignancies: expression, clinical pathology, prognostic 
Taweesak Tongtawee et al

markers and implications for chemotherapy. Curr Cancer Drug Targets, 5, 27-41.

Sheikh MS, Shao ZM, Hussain A, et al (1993). The p53-binding protein MDM2 gene is differentially expressed in human breast carcinoma. Cancer Res, 53, 3226-8.

Trotta R, Vignudelli T, Candini O, et al (2003). BCR/ABL activates mdm 2 mRNA translation via the La antigen. Cancer Cell, 3, 145-60.

Xiao ZX, Chen J, Levine AJ, et al (1995). Interaction between the retinoblastoma protein and the oncoprotein MDM2. Nature, 375, 694-8.

Wang X, Yang J, Ho B, et al (2009). Interaction of Helicobacter pylori with genetic variants in the MDM2 promoter

is associated with gastric cancer susceptibility in Chinese patients. Helicobacter, 14, 114-9. 